\title{
Preface for the ICEBE 2007 special section
}

\author{
S. C. Cheung - Hui Lei - Michael R. Lyu
}

Received: 3 March 2009 / Accepted: 17 March 2009 / Published online: 2 April 2009

(C) Springer-Verlag London Limited 2009

It is our greatest honor to edit this special section entitled "Selected Papers of ICEBE 2007", for the Journal of Service Oriented Computing and Applications. Our main objective is to disseminate inspiring work that addresses the problem of engineering e-Business systems. E-Business has emerged as an important computing paradigm in digital societies. The paradigm imposes many interesting challenges to both the research community and the industry. These challenges range from the reliable integration of e-services to trustworthy transactions of e-retailing. Addressing these challenges requires the cross fertilization of multiple disciplines, including databases, software engineering, networking, grid computing, security, machine intelligence and information systems.

This special section publishes two selected best papers presented in the IEEE International Conference on e-Business Engineering in 2007. The first paper presents an inspiring management framework to evaluate the trust and reputation of complex service-oriented applications. The second paper presents a novel entropy-based algorithm to provide reliable recommendation on B2B e-commerce services. We would like to thank all the referees who have worked hard to review each paper in a timely fashion, and provided authors with constructive comments on how to improve the work to its highest quality. We trust that you will find the articles of this special section interesting and informative.

Guest Editors

S. C. Cheung, Hui Lei, and Michael R. Lyu

March 2009

S. C. Cheung $(\varangle) \cdot$ H. Lei · M. R. Lyu

The Hong Kong University of Science and Technology,

Jiulong, Hong Kong

e-mail: scc@cs.ust.hk
S. C. Cheung is currently an Associate Professor of Computer Science at the Hong Kong University of Science and Technology. He is serving on the executive committee of the ACM SIGSOFT, the editorial board of IEEE Transactions on Software Engineering (TSE), the Journal of Computer Science and Technology (JCST), and International Journal of RF Technologies: Research and Application. His research interests include services computing, context-aware computing, object design patterns, software testing, ubiquitous computing, wireless sensor networks and RFID. He co-chaired the program committee of ICEBE'2007.

H. Lei received a Ph.D. in Computer Science from Columbia University. He is currently a Research Staff Member in the IBM T. J. Watson Research Center, where he manages the Messaging Systems department. He has served as conference co-chair or PC co-chair of MDM 2004, ICEBE 2005, Mobiquitous 2007, ICEBE 2007 and MDM 2008. His research interests include enterprise connectivity, business process management, service-oriented computing and mobile computing. He is a Senior Member of IEEE.

M. R. Lyu is a Professor in the Department of Computer Science and Engineering at the Chinese University of Hong Kong. He organized ISSRE'2001, PRDC'2005, and chaired or co-chaired the program committee of ISSRE'96, PRDC'99, WWW'2001, SRDS'2005, and ICEBE'2007. He has been on the Editorial Board of IEEE Transactions on Knowledge and Data Engineering, IEEE Transactions on Reliability, Journal of Information Science and Engineering, and Wiley Software Testing, Verification and Reliability Journal. Dr. Lyu is an IEEE Fellow, an AAAS Fellow, and a Croucher Senior Research Fellow. His research interests include software reliability, distributed systems, fault-tolerant computing, mobile and sensor networks, Web technologies, multimedia, and machine learning. 